\title{
Nano-scale Strain Mapping Using Advanced STEM with a Direct Electron Detector
}

\author{
V. B. Ozdol ${ }^{1}$, C. Gammer ${ }^{1}$, M.C. Sarahan ${ }^{2}$ and A. M. Minor ${ }^{1}$ \\ ${ }^{1 .}$ National Center for Electron Microscopy, Lawrence Berkeley National Laboratory, CA, USA and \\ Department of Materials Science and Engineering, University of California, Berkeley, CA, USA \\ 2. Gatan, Inc., Pleasanton, CA, USA
}

Elastic strain is an important materials property in semiconductor heterostructures, both controlling the crystal growth as well as directly affecting the electronic properties. Imaging and quantifying defect strain fields at the nanometer scale is therefore of great importance in these materials. Although HRTEM coupled with image processing algorithms such as GPA [1] has proven to be a very accurate method to measure strain, it still requires high quality specimens that are necessarily thin, which enhances strain relaxation. Recent advances in probe forming optics and detection systems allow STEM to be used for strain mapping both in imaging and diffraction mode.

The stability of modern microscopes equipped with high brightness electron source and probe correctors enables us to employ STEM for nanometer resolution strain analysis of relatively thicker TEM lamellas. Figure 1a displays an HRSTEM image of a GaAs/GaAsP superlattice structure extracted from a high efficiency photocathode [2] by means of FIB. The specimen was thinned to a final thickness of around 100 $\mathrm{nm}$ using low energy Ar ion milling. The $1 \mathrm{k} x 1 \mathrm{k}$ image was acquired on a probe-corrected FEI Titan (TEAM 0.5) operated at $300 \mathrm{kV}$ with a dwell time of $8 \mu \mathrm{s}$. Figure $1 \mathrm{~b}$ shows the enlargement of a stacking fault bounded by two $30^{\circ}$ partial dislocations. Figure $1 \mathrm{c}$ and $1 \mathrm{~d}$ displays color-coded two-dimensional inplane and out-of-plane strain maps calculated using GPA at $1 \mathrm{~nm}$ spatial resolution and $10^{-3}$ precision.

While high-resolution imaging is quite efficient for mapping strain fields induced by features limited in size, it becomes much more difficult if the strain fields extend beyond the field of view of a single HRSTEM image. To overcome this limitation we combined the nano-beam electron diffraction (NBED) technique with the capabilities of a direct detection camera. Figure 2a shows a HAADF image of an extended stacking fault originating at the $\mathrm{AlGaAsP} / \mathrm{GaAsP}$ interface. The image was acquired on an FEI Titan whose three independent condenser lenses allowed forming a sub-nanometer electron probe with a semi-convergence angle of $1.5 \mathrm{mrad}$ using a $40 \mu \mathrm{m}$ condenser aperture at $300 \mathrm{kV}$. For each individual pixel in the HAADF image (256px256p) NBED patterns were recorded simultaneously using the Gatan K2-IS direct detection camera at a frame rate of $400 \mathrm{f} / \mathrm{s}$. Figure $2 \mathrm{c}$ displays the virtual BF image extracted from a 4D data cube consisting of 65500 diffraction patterns. Color-coded 2D out-plane strain map shown in Figure 2d was calculated using a dedicated pattern recognition algorithm implemented in Digital Micrograph platform. This novel technique combines the large field of view of $250 \mathrm{~nm}$ with spatial resolution of $1 \mathrm{~nm}$ and $10^{-3}$ precision and relatively fast acquisition time of $160 \mathrm{~s}$.

In this contribution we will cover the guidelines for quantitative strain analysis using advanced STEM techniques including the optimization of illumination for imaging and diffraction mode as well as reliable reconstruction of the two-dimensional strain tensor from large data sets.

[1] M. J. Hÿtch, E. Snoeck, and R. Kilaas, Ultramicroscopy 74, 131 (1998)

[2] Jin, X. et al, Applied Physics Express 6, 015801 (2013)

[3] The authors acknowledge Prof. Xiuguang Jin at Nagoya University for providing the sample and

Gatan for use of a K2-IS camera for the experiments. 

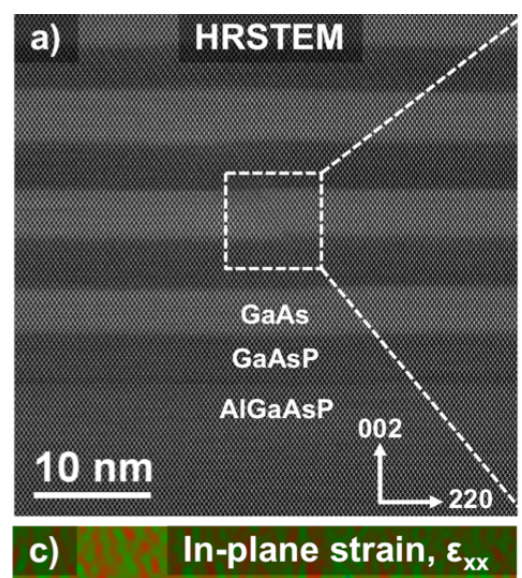

\section{$10 \mathrm{~nm}$}
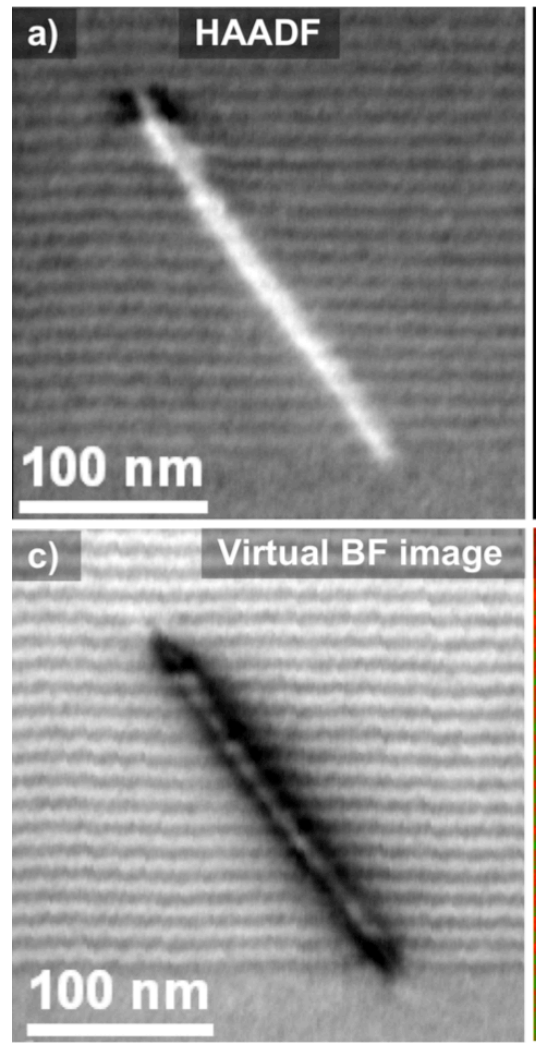
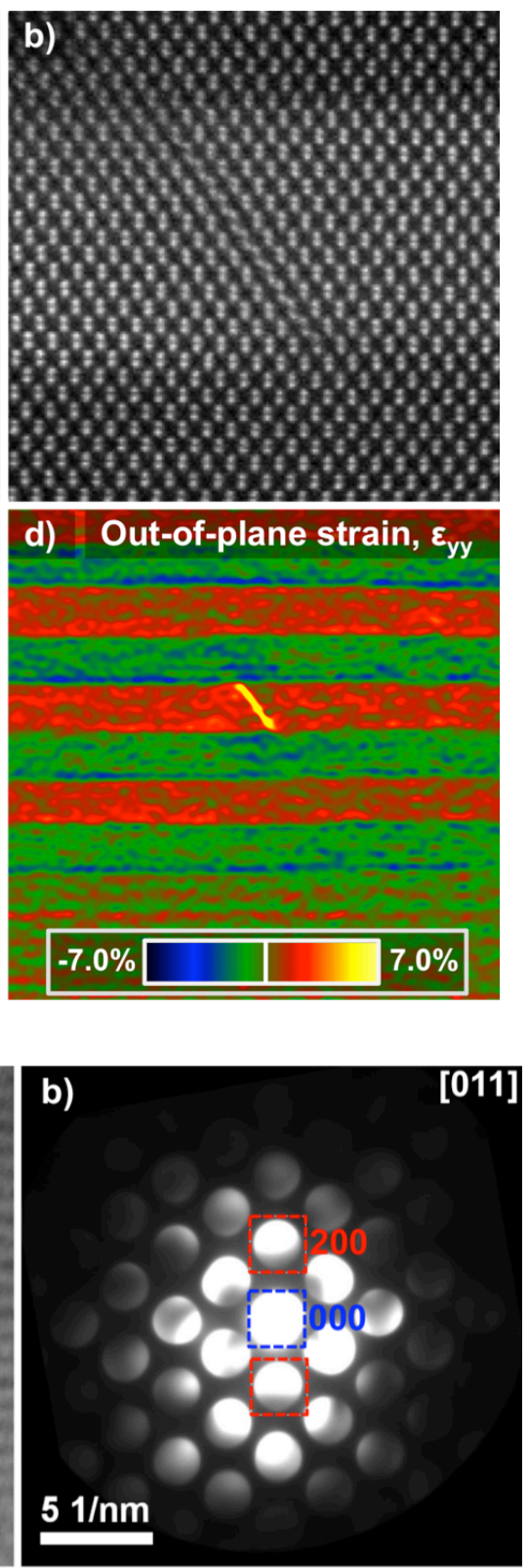

d) Out-of-plane strain, $\varepsilon_{y y}$

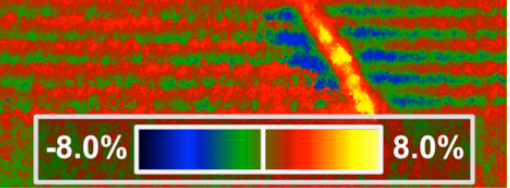

Figure 1. a) [110] HRSTEM image of a GaAs/GaAsP superlattice structure grown on an AlGaAsP virtual substrate. b) Enlargement of a stacking fault bounded by two $30^{\circ}$ partial dislocations. Two-dimensional inplane, c), and out-of-plane, d), strain maps calculated using GPA at $1 \mathrm{~nm}$ spatial resolution.

Figure 2. a) HAADF image of an extended stacking fault originating at the AlGaAsP/GaAsP interface. b) Integrated diffraction pattern of the superlattice structure along the [011] zone axis. c) Virtual BF image extracted from 4D data cube using the central beam as marked with blue in b). d) Out-ofplane strain map around the extended stacking fault reconstructed from the $4 \mathrm{D}$ data cube using (200) reflections as marked with red in b). The strain map combines the large field of view of $250 \mathrm{~nm}$ with spatial resolution of $1 \mathrm{~nm}$ and $10^{-3}$ precision. 